\section{P17.31 TIMED VAGINAL INSEMINATION AS A SAFER CONCEPTION METHOD FOR HIV-SERODISCORDANT COUPLES IN KISUMU, KENYA}

${ }^{1} \mathrm{O}$ Mmeje*, ${ }^{2} \mathrm{~B}$ Njoroge, ${ }^{2,3} \mathrm{P}$ Wekesa, ${ }^{4} \mathrm{MA}$ Guzé, ${ }^{5} \mathrm{~S}$ Shade, ${ }^{3,4} \mathrm{CR}$ Cohen. ${ }^{1}$ University of Michigan, Department of Obstetrics and Gynecology; ${ }^{2}$ Kenya Medical Research Institute (KEMRI), Centre for Microbiology Research; ${ }^{3}$ Family AIDS Care and Education Services (FACES); ${ }^{4}$ University of California, San Francisco, Department of Obstetrics, Gynecology \& Reproductive Sciences; ${ }^{5}$ University of California, San Francisco, Center for AIDS Prevention Studies

\subsection{6/sextrans-2015-052270.609}

Background Female positive/male negative $(++/ \hat{\gamma}-)$ HIV-serodiscordant couples desiring children have expressed an interest in safer conception interventions to reduce HIV transmission. Approximately $45 \%$ of HIV-infected women desire children and may choose to engage in condomless sex to achieve pregnancy. Without routinely available preconception counselling and safer conception reproductive services, $q+/ \hat{\delta}$ - HIV-serodiscordant couples who desire children represent a key population at risk of sexual HIV transmission.

Methods We conducted a prospective study of $q+/ \hat{\jmath}$ - HIV-serodiscordant couples desiring children in Kenya to evaluate the feasibility and efficacy of timed vaginal insemination (TVI). Eligible couples included female partners age 18-34 years with regular menses and HIV disclosure to male partners. Prior to TVI, couples were tested and treated for STIs, advised on and monitored for consistent condom use (i.e. evaluation for the presence of prostate specific antigen) and regular menses, and educated on TVI. The intervention included sexual intercourse with a condom and semen collection with a syringe for TVI during the fertile window for up to six menstrual cycles. Time to pregnancy with TVI was assessed with a Kaplan-Meier analysis.

Results Forty $++/ \delta^{-}$- HIV-serodiscordant couples were enrolled. Seventeen couples exited prior to TVI due to dissolution of the relationship $(\mathrm{n}=4)$, voluntary cessation of study participation $(\mathrm{n}=2)$, HIV seroconversion $(\mathrm{n}=2)$, irregular menses $(\mathrm{n}=2)$, or lost to follow-up $(n=7)$. Twenty-three couples $(57.5 \%)$ were introduced to TVI. At baseline, 17 (73.9\%) women reported ease with the TVI procedures. We observed eight pregnancies without HIV transmission, which resulted in six live births and two non-viable infants. Accounting for loss to follow-up, we estimate that $36 \%$ of women will become pregnant within 150 days of TVI initiation.

Conclusion Given the desire for children amongst HIV-affected couples, TVI may be acceptable and as effective in achieving pregnancy as natural conception while minimising the risk of HIV transmission.

Disclosure of interest statement This research was supported by a grant from the National Institutes of Health (NIH), University of California San Francisco-Gladstone Institute of Virology and Immunology Centre for AIDS Research (P30 AI027763). O. Mmeje was partly supported by NIH/T32-5T32AI06538803.

\section{P17.32 RISK FACTORS FOR NEVIRAPINE-ASSOCIATED RASH AND/OR HEPATOPATOTOXICITY AMONG HIV-INFECTED PATIENTS IN INDONESIA}

${ }^{1}$ ASR Pudjiati ${ }^{*},{ }^{1} \mathrm{H}$ Soebono, ${ }^{1} \mathrm{I}$ Dwiprahasto, ${ }^{2} \mathrm{Z}$ Djoerban. ${ }^{1}$ Faculty of Medicine- Universitas Gadjah Mada, Indonesia; ${ }^{2}$ Faculty of Medicine- Universitas Indonesia, Indonesia

10.1136/sextrans-2015-052270.610
Introduction Nevirapine (NVP) is commonly used as a component of first-line antiretroviral therapy in Indonesia. We aimed to determine the risk factors for NVP-associated rash and/or heaptotoxicity among HIV-infected patients in Indonesia.

Methods A case-control study was conducted in HIV-infected patients who developed rash after taking NVP or increasing level of transaminase enzim (case) and those who did not have rash or increasing level of transaminase enzim (control).

Results A total of 149 patients with a mean (SD) age of 35.2 (10.2) years; $84(56.4 \%)$ male, 56 (37.6\%) female and 9 (6.0\%) transgender were included in the study. Mean body weight (SD) was $56.7(38.8) \mathrm{kg}$. Of all, $9(6.0 \%)$ patients had a history of AIDS-defining illness and $12(8.1 \%)$ patients had history of drug allergy. Mean CD4 cell counts at the time of NVP initiation was $147.3(2-615)$ cells/mm3. There were 49 patients in case group and 100 patients in control group. In case group,18.4\%, 73.9\%, $18.4 \%$ and $18.4 \%$ of patients developed grade $1,2,3$, and 4 of rash, $57.1 \%, 21.4 \%, 7.1 \%$ and $14.3 \%$ of patients developed grade $1,2,3$, and 4 of hepatotoxicity, respectively. Mean time to develop rash was 19.4 (5-52) days. By logistic regression, history of drug allergy (OR, 4.20; 95\% CI, 0.64-27.84) body weight (OR, 1.15; 95\% CI, 0.72-1.82), CD4 cells counts (OR, 0.85; 95\% CI, 0.54-1.35), and AIDS-defining illness (OR, 0.99; 95\% CI, 0.38-2.56) were not significantly associated with nevirapineassociated rash and/or hepatotoxicity among HIV-infected patients in Indonesia.

Conclusion In Indonesia settings where patients were initiated NVP, history of drug allergy, lower body weight, and higher CD4 cell count are not the risk factors for NVP-associated rash and/or hepatotoxicity.

Disclosure of interest statement Authors declare that there is no conflict of interest regarding the publication of the paper.

\section{P17.33 EFAVIRENZ-ASSOCIATED SERIOUS ADVERSE REACTIONS: AN ANALYSIS OF THE THAI ADVERSE EVENTS REPORTING SYSTEM}

Pakawadee Sriphiromya*. Ministry of Public Health, Thailand

\subsection{6/sextrans-2015-052270.611}

Introduction Voluntary spontaneous reporting system has been used as a major method to report ADRs in Thailand. Efavirenz is in the NNRTI class, combined use with other antiretroviral (ARV) to treat the infection. Efavirenz is metabolised by liver and various adverse drug reactions (ADRs) can be found when used it for long term or with other drugs. Explore the distribution and determinant of ADR associated with ARV in Thai population is not well established but crucial for improve patient care and generate risk minimization in both population and individual level.

Objective The Thai adverse event reports from 2010 to 2014 were reviewed to assess serious adverse events induced by efavirenz.

Methods Thai adverse event reports data were assessed by elimination of duplicated records as well as adjustments to standardise drug names, reports involving efavirenz were analysed. Exposure variables are efavirenz used. Outcome variables are serious ADRs which happened within the study period. Other important variable such as sex, co morbidity, multiple drugs used are evaluated. Hepatitis, exfoliative dermatitis, and Stevens Johnson syndrome were focused on as serious adverse events. Signals 